\title{
A POLARIZATION OUTBURST IN THE T TAURI STAR UY AURIGAE
}

\author{
F. Ménard and P. Bastien \\ Département de Physique and Observatoire du mont Mégantic \\ Université de Montréal \\ B.P. 6128, Succ. A, Montréal, Québec H3C 3J7 Canada
}

During the course of a monitoring programme of the linear polarization of various $T$ Tauri stars, UY Aur was observed to undergo a strong polarization burst between 1984 October and 1985 January. The linear polarization rised from about $1.5 \%$ early in 1984 to a maximum of $7.6 \%$ in 1984 October and declined back to the earlier value after 3 to 4 months. It is the first time that such a large increase in linear polarization is reported in a $\mathrm{T}$ Tauri star. However the interpretation is complicated by the fact that UY Aur is a visual binary with a separation of 0.8 and both components were included in the measurements.

The linear polarization in $\mathrm{T}$ Tauri stars is due mostly to scattering of light from the stars and their immediate line emitting regions by grains outside these inner regions (Bastien and Landstreet 1979). This is supported in the case of UY Aur by a strong infrared excess (e.g. V-L $=6.50$, Rydgren and Vrba (1983)) and also by its IRAS detection at $12,25,60$ and $100 \mu \mathrm{m}$. Assuming single scattering, one can compute the wavelength dependence of polarization $P(\lambda)$ to be expected from a distribution of various grain particles. No single grain composition and grain radius could fit the observed $P(\lambda)$, see Figure 1 , near the end of the polarization burst. A good fit can be obtained, to within a scale factor, with of mixture of spherical amorphous carbon grains of 0.16 and $0.23 \mu \mathrm{m}$ in radius. The details of the fit can be found in Ménard (1986).

\section{REFERENCES}

Bastien, P., and Landstreet, J.D.: 1979, Astrophys. J. (Letters) 222, L137.

Ménard, F.: 1986, M. Sc. Thesis, Univ. of Montréa1.

Rydgren, A.E., and Vrba, F.J.: 1983, Astron. J. $\underline{8} 8$ (Z) 1017 . 


\section{UY AURIGAE}

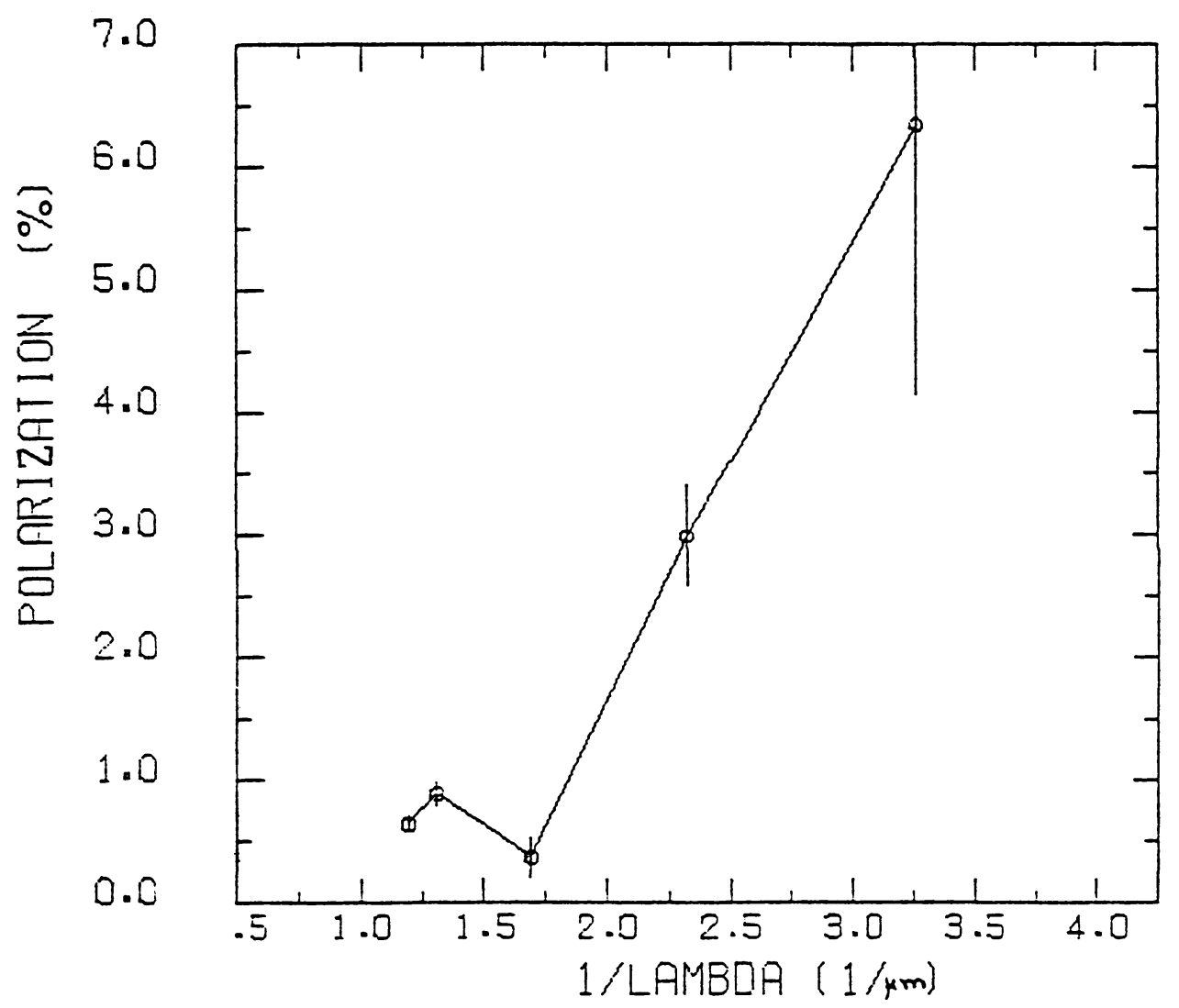

FIGURE 1 Observed polarization curve of UY Aurigae. The measurements were taken on 1985 January 31 st. 